\title{
Positive solutions for a class of nonhomogeneous Kirchhoff-Schrödinger-Poisson systems
}

Hongxia Shi ${ }^{1 *}$ (D)

"Correspondence:
shihongxia5617@163.com
'School of Mathematics and
Computational Science, Hunan First
Normal University, Changsha,
P.R. China

\section{Abstract}

This paper deals with the following generalized nonhomogeneous Kirchhoff-Schrödinger-Poisson system:

$$
\begin{cases}\left(a+\int_{\mathbb{R}^{3}}|\nabla u|^{2}+b \int_{\mathbb{R}^{3}} u^{2}\right)(-\Delta u+b u)+q \phi f(u)=g(u)+h(x), & \text { in } \mathbb{R}^{3}, \\ -\Delta \phi=2 q F(u), & \text { in } \mathbb{R}^{3},\end{cases}
$$

where $a>0, b \geq 0$ are constants, $a \geq 0$ is a parameter, and $F(t)=\int_{0}^{t} f(s) \mathrm{d} s$. Under some appropriate assumptions on $g(u)$ and $h(x)$, the existence of two positive radial solutions is proved by applying Ekeland's variational principle and the mountain pass theorem.

MSC: $35 J 50 ; 35 J 60$

Keywords: Kirchhoff-Schrödinger-Poisson systems; Variational methods; Cut-off functional; Pohozaev type identity

\section{Introduction and main results}

In this paper, we consider the existence of multiple positive solutions for the following generalized nonhomogeneous Kirchhoff-Schrödinger-Poisson system:

$$
\begin{cases}\left(a+\int_{\mathbb{R}^{3}}|\nabla u|^{2}+b \int_{\mathbb{R}^{3}} u^{2}\right)(-\Delta u+b u)+q \phi f(u)=g(u)+h(x), & \text { in } \mathbb{R}^{3}, \\ -\Delta \phi=2 q F(u), & \text { in } \mathbb{R}^{3},\end{cases}
$$

where $a>0, b \geq 0$ are constants, $q \geq 0$ is a parameter, $F(t)=\int_{0}^{t} f(s) \mathrm{d} s, f$ and $h$ satisfy the following hypotheses:

(f) $f \in C\left(\mathbb{R}^{+}, \mathbb{R}^{+}\right)$and there exists $C>0$ such that $f(t) \leq C\left(|t|+|t|^{\alpha}\right)$ for all $t \in \mathbb{R}$, where $\alpha \in(2,4)$;

$\left(h_{1}\right) 0 \leq h(x) \in L^{2}\left(\mathbb{R}^{3}\right) \cap C^{1}\left(\mathbb{R}^{3}\right)$ and $0 \not \equiv h(x)=h(|x|)$;

$\left(h_{2}\right)\|h\|_{2} \leq m_{p}$ with $m_{p}=\frac{a(p-2)}{4 \gamma_{2}(p-1)}\left(\frac{a p}{\left.4 m(p-1) \gamma_{p}^{p}\right)^{1 /(p-2)}}\right.$, where $p \in(2,6)$ and $\gamma_{p}$ is the Sobolev embedding constant of $H^{1}\left(\mathbb{R}^{3}\right) \hookrightarrow L^{p}\left(\mathbb{R}^{3}\right), m$ is a constant depending on $\gamma_{2}$ which will be introduced in Lemma 3.1;

$\left(h_{3}\right)\langle\nabla h(x), x\rangle \in L^{2}\left(\mathbb{R}^{3}\right)$ and $\langle\nabla h(x), x\rangle \geq 0$, where $\langle\cdot, \cdot \cdot\rangle$ denotes the usual inner product.

\section{Springer}


When $q=0$, system (1.1) reduces to the following Kirchhoff-type equation:

$$
\left(a+\int_{\mathbb{R}^{3}}|\nabla u|^{2}+b \int_{\mathbb{R}^{3}} u^{2}\right)(-\Delta u+b u)=g(u)+h(x), \quad \text { in } \mathbb{R}^{3} .
$$

In recent years, the following Dirichlet problem of Kirchhoff-type on a bounded domain $\Omega \subset \mathbb{R}^{N}$

$$
\begin{cases}-\left(a+b \int_{\Omega}|\nabla u|^{2}\right) \Delta u+V(x) u=f(x, u), & \text { in } \Omega, \\ u=0, & \text { on } \partial \Omega,\end{cases}
$$

has been studied extensively by many researchers. With the aid of variational methods, for various conditions of the potential $V(x)$ and the nonlinearity $f(x, u)$, the existence, nonexistence, and multiplicity results for problem (1.3) have been investigated in the literature, one can see $[1,2,6,8,12,17,22]$ and the references therein. There are also many works on the existence and multiplicity results for system (1.3) on unbounded domains. More precisely, Duan and Huang [7] dealt with problem (1.3) with sublinear case, and the existence of infinitely many solutions for the problem has been established by using the genus properties in critical point theory. Wu [27] studied the existence of nontrivial solutions and infinitely many high energy solutions for problem (1.3) by using a symmetric mountain pass theorem. Liu and He [15] also studied the existence of infinitely many high energy solutions for superlinear Kirchhoff problem (1.3) by a variant version of the fountain theorem. For more related topics, we refer the readers to $[9,14,23,28-30]$ and the references therein.

Very recently, Li et al. [14] studied the existence of at least one positive radial solution to the following nonlinear homogeneous Kirchhoff-type equation:

$$
\left(a+\lambda \int_{\mathbb{R}^{N}}|\nabla u|^{2}+\lambda b \int_{\mathbb{R}^{N}} u^{2}\right)(-\Delta u+b u)=f(u), \quad \text { in } \mathbb{R}^{N},
$$

where $N \geq 3, \lambda \geq 0$ is a parameter. Their result can be regarded as an extension of a classical result for the semilinear equation

$$
-\Delta u+b u=f(u), \quad \text { in } \mathbb{R}^{N},
$$

to the nonlinear Kirchhoff-type equation (1.4). The more general semilinear Schrödinger equation (1.5) with $b=V(x)$ has been studied by many researchers under various stipulations, one can see $[16,24,25]$ and the references therein.

On the other hand, the well-known Schrödinger-Poisson system

$$
\begin{cases}-\Delta u+V(x) u+q \phi u=f(x, u), & \text { in } \mathbb{R}^{3}, \\ -\Delta \phi=q u^{2}, & \text { in } \mathbb{R}^{3},\end{cases}
$$

also known as the nonlinear Schrödinger-Maxwell system, and the similar systems arise in many mathematical physics contexts, such as in quantum electrodynamics, to describe the interaction between a charge particle interacting with the electromagnetic field, and also in semiconductor theory, in nonlinear optics, and in plasma physics (see [5] for more 
details on the physics aspects). Many papers deal with problem (1.6) by using the modern variational method and the critical point theory under various assumptions on the potential $V(x)$ and the nonlinearity $f(x, u)$; see [19-21] and the references therein. Very recently, there have also been many authors who investigated the generalized Schrödinger-Poisson system on bounded domains or on unbounded domains, see [3, 4, 13, 31]. In [13] the authors considered the following system:

$$
\begin{cases}-\Delta u+u+q \phi f(u)=g(u), & \text { in } \mathbb{R}^{3}, \\ -\Delta \phi=2 q F(u), & \text { in } \mathbb{R}^{3} .\end{cases}
$$

They proved that there exists $q_{0}>0$ such that the system has at least a positive radial solution for $q \in\left[0, q_{0}\right)$.

By using the symmetric mountain pass theorem, the authors in [32] studied the following Kirchhoff-Schrödinger-Poisson system:

$$
\begin{cases}\left(a+b \int_{\mathbb{R}^{3}}\left[|\nabla u|^{2}+V(x) u^{2}\right]\right)[-\Delta u+V(x) u]+\lambda l(x) \phi u=f(x, u), & \text { in } \mathbb{R}^{3}, \\ -\Delta \phi=\lambda l(x) u^{2}, & \text { in } \mathbb{R}^{3},\end{cases}
$$

where constants $a>0, b \geq 0$, and $\lambda \geq 0$. They proved the existence of infinitely many solutions assuming that $f$ has sublinear growth in $u$.

Motivated by the above facts, in this paper, we consider the more general nonhomogeneous Kirchhoff-Schrödinger-Poisson system (1.1). The aim of this paper is to prove the existence and multiplicity of positive radial solutions for system (1.1) when $f$ is subcritical and $g$ is superlinear at infinity. To the best of our knowledge, there have been no works concerning this case up to now. Compared to the aforementioned results, our result extends these results to some extent.

In this paper, since we are concerned with the existence of positive solutions to (1.1), we assume that $f(t)=g(t)=0$ for $t<0$. And the following assumptions will be used in this paper.

$\left(g_{1}\right) g \in C\left(\mathbb{R}^{+}, \mathbb{R}^{+}\right)$and there exists $C>0$ such that $g(t) \leq C\left(1+|t|^{p-1}\right)$ for all $t \in \mathbb{R}$, where $p \in(2,6)$;

$\left(g_{2}\right) \lim _{t \rightarrow 0} g(t) / t=0$;

$\left(g_{3}\right) \lim _{t \rightarrow \infty} g(t) / t^{3}=\infty$.

The main results of the present paper can be described as follows.

Theorem 1.1 Assume that $(f),\left(h_{1}\right)-\left(h_{3}\right)$, and $\left(g_{1}\right)-\left(g_{3}\right)$ hold. Then there exists $q_{0}>0$ such that problem (1.1) has at least two positive radial solutions $(u, \phi) \in H^{1}\left(\mathbb{R}^{3}\right) \times \mathcal{D}^{1,2}\left(\mathbb{R}^{3}\right)$ for all $0 \leq q<q_{0}$ and $0 \leq b \leq 1 / 4 \gamma_{2}^{2}$.

According to Theorem 3.4 in Sect. 3, we have the following result.

Remark 1.2 Assume that $(f),\left(h_{1}\right)-\left(h_{2}\right)$, and $\left(g_{1}\right)-\left(g_{3}\right)$ hold. Then, for any $q \geq 0$, problem (1.1) has at least one positive radial solution $(u, \phi) \in H^{1}\left(\mathbb{R}^{3}\right) \times \mathcal{D}^{1,2}\left(\mathbb{R}^{3}\right)$.

This paper is organized as follows. In Sect. 2, we give the variational framework to our problem. Section 3 is devoted to proving the existence of a local minimum around the 
origin with negative energy. In Sect. 4, the existence of mountain-pass type critical point is obtained.

\section{Variational setting and preliminaries}

In this section, we assume that $(f),\left(h_{1}\right),\left(g_{1}\right)$, and $\left(g_{2}\right)$ hold, then we study the variational framework of (1.1). Firstly, we give the following notations:

- $H^{1}\left(\mathbb{R}^{3}\right)$ is the usual Sobolev space equipped with the inner product and norm

$$
\langle u, v\rangle=\int_{\mathbb{R}^{3}}(\nabla u \cdot \nabla v+b u v) \mathrm{d} x, \quad\|u\|=\langle u, u\rangle^{1 / 2} .
$$

- $L^{s}\left(\mathbb{R}^{3}\right)(1 \leq s \leq+\infty)$ is a Lebesgue space whose norm is denoted by $\|\cdot\|_{s}$.

- Let $\mathcal{D}^{1,2}\left(\mathbb{R}^{3}\right):=\left\{u \in L^{6}\left(\mathbb{R}^{3}\right):|\nabla u| \in L^{2}\left(\mathbb{R}^{3}\right)\right\}$ be the Sobolev space with the norm $\|u\|_{\mathcal{D}^{1,2}}^{2}:=\int_{\mathbb{R}^{3}}|\nabla u|^{2} \mathrm{~d} x$.

- $C$ and $C_{i}$ denote various positive constants, which may vary from line to line.

- The strong (respectively weak) convergence is denoted by $\rightarrow$ (respectively $\rightarrow$ ).

- $B_{\rho}(0)$ denotes a ball centered at the origin with radius $\rho>0$.

- Let $S$ be the best constant of the Sobolev embedding $\mathcal{D}^{1,2}\left(\mathbb{R}^{3}\right) \hookrightarrow L^{6}\left(\mathbb{R}^{3}\right)$, which is given by

$$
S=\inf _{u \in \mathcal{D}^{1,2}\left(\mathbb{R}^{3}\right) \backslash\{0\}} \frac{\|u\|_{\mathcal{D}^{1,2}}^{2}}{\|u\|_{6}^{2}} .
$$

Now we have that the embedding $H^{1}\left(\mathbb{R}^{3}\right) \hookrightarrow L^{s}\left(\mathbb{R}^{3}\right)$ is continuous for $s \in[2,6]$, and there exists $\gamma_{s}>0$ such that

$$
\|u\|_{s} \leq \gamma_{s}\|u\|, \quad \forall u \in H^{1}\left(\mathbb{R}^{3}\right)
$$

Let $H=H_{r}^{1}\left(\mathbb{R}^{3}\right)$ be the subspace of $H^{1}\left(\mathbb{R}^{3}\right)$ containing only the radial functions. Then, due to the result of [26], the embedding $H \hookrightarrow L^{s}\left(\mathbb{R}^{3}\right)$ is compact for $s \in(2,6)$.

By virtue of the variational nature of problem (1.1), its weak solutions $(u, \phi) \in H^{1}\left(\mathbb{R}^{3}\right) \times$ $\mathcal{D}^{1,2}\left(\mathbb{R}^{3}\right)$ are critical points of the $C^{1}$ functional $J: H^{1}\left(\mathbb{R}^{3}\right) \times \mathcal{D}^{1,2}\left(\mathbb{R}^{3}\right) \rightarrow \mathbb{R}$ defined by

$$
\begin{aligned}
J(u, \phi)= & \frac{a}{2}\|u\|^{2}+\frac{1}{4}\|u\|^{4}-\frac{1}{4} \int_{\mathbb{R}^{3}}|\nabla \phi|^{2} \mathrm{~d} x+q \int_{\mathbb{R}^{3}} F(u) \phi \mathrm{d} x \\
& -\int_{\mathbb{R}^{3}} G(u) \mathrm{d} x-\int_{\mathbb{R}^{3}} h u \mathrm{~d} x,
\end{aligned}
$$

where $G(u)=\int_{0}^{u} g(s) \mathrm{d} s$. By condition $(f)$ and the Lax-Milgram theorem, for every $u \in$ $H^{1}\left(\mathbb{R}^{3}\right)$, there exists unique $\phi=\phi_{u} \in \mathcal{D}^{1,2}\left(\mathbb{R}^{3}\right)$ satisfying $-\Delta \phi=2 q F(u)$. Similar to the argument in [13], we can derive that the function $\phi_{u}$ has the following properties.

Lemma 2.1 ([13]) For every $u \in H^{1}\left(\mathbb{R}^{3}\right)$, we have

(i) $\left\|\phi_{u}\right\|_{\mathcal{D}^{1,2}}^{2}=2 q \int_{\mathbb{R}^{3}} F(u) \phi_{u} \mathrm{~d} x$;

(ii) $\phi_{u} \geq 0$;

(iii) $\left\|\phi_{u}\right\|_{\mathcal{D}^{1,2}} \leq q C\left(\|u\|^{2}+\|u\|^{\alpha+1}\right)$;

(iv) $\int_{\mathbb{R}^{3}} F(u) \phi_{u} \mathrm{~d} x \leq q C_{1}\left(\|u\|^{4}+\|u\|^{2(\alpha+1)}\right)$;

(v) if $u$ is a radial function, then $\phi_{u}$ is radial too. 
According to Lemma 2.1 and (2.1), we can prove that $(u, \phi) \in H^{1}\left(\mathbb{R}^{3}\right) \times \mathcal{D}^{1,2}\left(\mathbb{R}^{3}\right)$ is a solution of (1.1) if and only if $u \in H^{1}\left(\mathbb{R}^{3}\right)$ is a critical point of the one variable functional defined as

$$
J_{q}(u)=\frac{a}{2}\|u\|^{2}+\frac{1}{4}\|u\|^{4}+\frac{q}{2} \int_{\mathbb{R}^{3}} F(u) \phi_{u} \mathrm{~d} x-\int_{\mathbb{R}^{3}} G(u) \mathrm{d} x-\int_{\mathbb{R}^{3}} h u \mathrm{~d} x .
$$

It is easy to check that $J_{q}: H \rightarrow \mathbb{R}$ is well defined and is class of $C^{1}$, and we have

$$
\begin{aligned}
\left\langle J_{q}^{\prime}(u), v\right\rangle= & a\langle u, v\rangle+\|u\|^{2}\langle u, v\rangle+q \int_{\mathbb{R}^{3}} f(u) \phi_{u} v \mathrm{~d} x \\
& -\int_{\mathbb{R}^{3}} g(u) v \mathrm{~d} x-\int_{\mathbb{R}^{3}} h v \mathrm{~d} x, \quad \forall u, v \in H .
\end{aligned}
$$

In order to obtain the existence of mountain-pass type critical point in Sect. 4, the boundedness of Palais-Smale sequences is crucial. But the standard arguments used to prove the boundedness of Palais-Smale sequences do not work. To overcome the difficulty, following [11], we use a cut-off function $\chi \in C\left(\mathbb{R}_{+},[0,1]\right)$ satisfying

$$
\begin{cases}\chi(t)=1, & t \in[0,1 / 2], \\ \chi(t)=0, & t \in[1, \infty], \\ \left\|\chi^{\prime}\right\|_{\infty} \leq 4, & \end{cases}
$$

and study the following modified functional $J_{q}^{T}: H \rightarrow \mathbb{R}$ defined by

$$
J_{q}^{T}(u)=\frac{a}{2}\|u\|^{2}+\frac{1}{4}\|u\|^{4}+\frac{q}{2} h_{T}(u) \int_{\mathbb{R}^{3}} F(u) \phi_{u} \mathrm{~d} x-\int_{\mathbb{R}^{3}} G(u) \mathrm{d} x-\int_{\mathbb{R}^{3}} h u \mathrm{~d} x, \quad \forall u \in H
$$

where $h_{T}(u)=\chi\left(\frac{\|u\|^{2}}{T^{2}}\right)$. In the following, we discuss the existence of critical points of $J_{q}^{T}$. In fact, for $T>0$ sufficiently large and $q$ sufficiently small, we can find a critical point of $J_{q}^{T}$ such that $\|u\| \leq T / \sqrt{2}$, hence $u$ is also a critical point of $J_{q}$.

In Sect. 4, we use the method based on the "monotonicity trick" introduced by Struwe in [18], which has been successfully used to handle many homogeneous elliptic problems. Now we recall the following result.

Theorem $2.2([10])$ Let $(X,\|\cdot\|)$ be a Banach space and $I \subset \mathbb{R}_{+}$be an interval. Consider the family of $C^{1}$ functionals on $X$ :

$$
J_{\lambda}(u)=A(u)-\lambda B(u), \quad \lambda \in I,
$$

with B nonnegative and either $A(u) \rightarrow \infty$ or $B(u) \rightarrow \infty$ as $\|u\| \rightarrow \infty$. We assume that there are two points $v_{1}, v_{2} \in X$ such that

$$
c_{\lambda}=\inf _{\gamma \in \Gamma_{\lambda}} \max _{t \in[0,1]} J_{\lambda}(\gamma(t))>\max \left\{J_{\lambda}\left(v_{1}\right), J_{\lambda}\left(v_{2}\right)\right\}, \quad \forall \lambda \in I
$$

where

$$
\Gamma_{\lambda}=\left\{\gamma \in C([0,1], X): \gamma(0)=v_{1}, \gamma(1)=v_{2}\right\}
$$


Then, for almost every $\lambda \in I$, there is a sequence $\left\{u_{n}\right\} \subset X$ such that

(i) $\left\{u_{n}\right\}$ is bounded;

(ii) $J_{\lambda}\left(u_{n}\right) \rightarrow c_{\lambda}$;

(iii) $J_{\lambda}^{\prime}\left(u_{n}\right) \rightarrow 0$ in the dual space $X^{-1}$ of $X$.

\section{Existence of solution $\boldsymbol{u}_{0}$ with negative energy}

In this section, we prove that, for any $q \geq 0$, system (1.1) has a positive radial solution with negative energy. With the aid of Ekeland's variational principle, this solution is obtained by seeking a local minimum of the energy functional $J_{q}$. Now we give some useful lemmas that will be used later.

Lemma 3.1 Assume that $(f),\left(h_{1}\right),\left(h_{2}\right),\left(g_{1}\right),\left(g_{2}\right)$, and $\left(g_{3}\right)$ hold. Then there exist $\rho, \alpha>0$ such that, for all $q \geq 0,\left.J_{q}\right|_{\|u\|=\rho}(u) \geq \alpha$.

Proof By conditions $\left(g_{1}\right)$ and $\left(g_{2}\right)$, for $\frac{a}{2 \gamma_{2}^{2}}$, there exists $m>0$ such that

$$
g(u) \leq \frac{a}{2 \gamma_{2}^{2}}|u|+m|u|^{p-1}, \quad \forall u \in \mathbb{R},
$$

and

$$
G(u) \leq \frac{a}{4 \gamma_{2}^{2}} u^{2}+\frac{m}{p}|u|^{p}, \quad \forall u \in \mathbb{R} .
$$

Then, for all $q \geq 0$ and $u \in H$, by Lemma 2.1, Hölder's inequality, and Sobolev's embedding theorem, we have

$$
\begin{aligned}
J_{q}(u) & =\frac{a}{2}\|u\|^{2}+\frac{1}{4}\|u\|^{4}+\frac{q}{2} \int_{\mathbb{R}^{3}} F(u) \phi_{u} \mathrm{~d} x-\int_{\mathbb{R}^{3}} G(u) \mathrm{d} x-\int_{\mathbb{R}^{3}} h u \mathrm{~d} x \\
& \geq \frac{a}{2}\|u\|^{2}-\int_{\mathbb{R}^{3}} G(u) \mathrm{d} x-\int_{\mathbb{R}^{3}} h u \mathrm{~d} x \\
& \geq \frac{a}{2}\|u\|^{2}-\int_{\mathbb{R}^{3}}\left(\frac{a}{4 \gamma_{2}^{2}} u^{2}+\frac{m}{p}|u|^{p}\right) \mathrm{d} x-\int_{\mathbb{R}^{3}} h u \mathrm{~d} x \\
& \geq\|u\|\left(\frac{a}{4}\|u\|-\frac{m}{p} \gamma_{p}^{p}\|u\|^{p-1}-\gamma_{2}\|h\|_{2}\right) .
\end{aligned}
$$

Set

$$
\eta(t)=\frac{a}{4} t-\frac{m}{p} \gamma_{p}^{p} t^{p-1}, \quad t \geq 0 .
$$

Since $p \in(2,6)$, by direct calculation, we see that

$$
\max _{t \geq 0} \eta(t)=\eta(\rho)=\frac{a(p-2)}{4(p-1)} \rho,
$$

where

$$
\rho=\left(\frac{a p}{4 m(p-1) \gamma_{p}^{p}}\right)^{1 /(p-2)} .
$$


Then it follows from (3.3) that, if $\gamma_{2}\|h\|_{2}<\eta(\rho)$, i.e., $\|h\|_{2}<\gamma_{2}^{-1} \eta(\rho):=m_{p}$, there exists $\alpha=\rho\left(\eta(\rho)-\gamma_{2}\|h\|_{2}\right)>0$ such that $\left.J_{q}\right|_{\|u\|=\rho}(u) \geq \alpha$, where $m_{p}=\frac{a(p-2)}{4 \gamma_{2}(p-1)}\left(\frac{a p}{4 m(p-1) \gamma_{p}^{p}}\right)^{1 /(p-2)}$.

Lemma 3.2 Assume that $(f),\left(h_{1}\right)$, and $\left(g_{1}\right)$ hold. Then, for any $q \geq 0$,

$$
c_{0}=\inf \left\{J_{q}(u): u \in \bar{B}_{\rho}\right\}<0,
$$

where $\rho$ is given by Lemma 3.1 and $\bar{B}_{\rho}=\{u \in H$ and $\|u\| \leq \rho\}$.

Proof By $\left(h_{1}\right)$, we can choose a function $\varphi \in H$ such that $\int_{\mathbb{R}^{3}} h(x) \varphi \mathrm{d} x>0$. By Lemma 2.1, for $t>0$ small enough, we obtain

$$
\begin{aligned}
J_{q}(t \varphi) & =\frac{a t^{2}}{2}\|u\|^{2}+\frac{t^{4}}{4}\|\varphi\|^{4}+\frac{q}{2} \int_{\mathbb{R}^{3}} F(t \varphi) \phi_{t \varphi} \mathrm{d} x-\int_{\mathbb{R}^{3}} G(t \varphi) \mathrm{d} x-t \int_{\mathbb{R}^{3}} h \varphi \mathrm{d} x \\
& \leq \frac{a t^{2}}{2}\|u\|^{2}+\frac{t^{4}}{4}\|\varphi\|^{4}+\frac{q^{2}}{2} C_{1}\left(t^{4}\|\varphi\|^{4}+t^{2(1+\alpha)}\|\varphi\|^{2(1+\alpha)}\right)-t \int_{\mathbb{R}^{3}} h \varphi \mathrm{d} x \\
& <0
\end{aligned}
$$

which shows that $c_{0}=\inf \left\{J_{q}(u): u \in \bar{B}_{\rho}\right\}<0$.

Lemma 3.3 Assume that $(f),\left(h_{1}\right),\left(g_{1}\right),\left(g_{2}\right)$, and $\left(g_{3}\right)$ hold. If $\left\{u_{n}\right\}$ is a bounded PalaisSmale sequence of $J_{q}$, then $\left\{u_{n}\right\}$ has a convergent subsequence in $H$.

Proof Since $\left\{u_{n}\right\}$ is a bounded (PS) sequence of $J_{q}$, then $J_{q}\left(u_{n}\right)$ is bounded, $J_{q}^{\prime}\left(u_{n}\right) \rightarrow 0$ in $H^{-1}$, where $H^{-1}$ is the dual space of $H$. We may assume that, up to a subsequence,

$$
\begin{array}{ll}
u_{n} \rightarrow u & \text { in } H, \\
u_{n} \rightarrow u & \text { in } L^{s}\left(\mathbb{R}^{3}\right), s \in(2,6), \\
u_{n} \rightarrow u & \text { a.e. on } \mathbb{R}^{3} .
\end{array}
$$

By $\left(g_{1}\right)$ and $\left(g_{2}\right)$, for any $\varepsilon>0$, there exists $C_{\varepsilon}>0$ such that

$$
g(u) \leq \varepsilon|u|+C_{\varepsilon}|u|^{p-1}, \quad \forall u \in \mathbb{R}
$$

then

$$
\begin{aligned}
\left|\int_{\mathbb{R}^{3}} g\left(u_{n}\right)\left(u_{n}-u\right) \mathrm{d} x\right| & \leq \int_{\mathbb{R}^{3}}\left[\varepsilon\left|u_{n}\right|+C_{\varepsilon}\left|u_{n}\right|^{p-1}\right]\left|u_{n}-u\right| \mathrm{d} x \\
& \leq \varepsilon\left\|u_{n}\right\|_{2}\left\|u_{n}-u\right\|_{2}+C_{\varepsilon}\left\|u_{n}\right\|_{p}^{p-1}\left\|u_{n}-u\right\|_{p} \\
& \leq \varepsilon\left\|u_{n}\right\|\left\|u_{n}-u\right\|+C_{\varepsilon} \gamma_{p}^{p-1}\left\|u_{n}\right\|^{p-1}\left\|u_{n}-u\right\|_{p} .
\end{aligned}
$$

It follows that

$$
\int_{\mathbb{R}^{3}} g\left(u_{n}\right)\left(u_{n}-u\right) \mathrm{d} x \rightarrow 0, \quad n \rightarrow \infty
$$


By a similar argument, we have

$$
\int_{\mathbb{R}^{3}} g(u)\left(u_{n}-u\right) \mathrm{d} x \rightarrow 0, \quad n \rightarrow \infty .
$$

By condition $(f)$ and Hölder's inequality, we obtain

$$
\begin{aligned}
& \left|\int_{\mathbb{R}^{3}} f\left(u_{n}\right) \phi_{u_{n}}\left(u_{n}-u\right) \mathrm{d} x\right| \\
& \quad \leq C \int_{\mathbb{R}^{3}}\left[\left|u_{n}\right|+\left|u_{n}\right|^{\alpha}\right] \phi_{u_{n}}\left|u_{n}-u\right| \mathrm{d} x \\
& \quad \leq C\left[\left\|\phi_{u_{n}}\right\| 6\left\|u_{n}\right\|_{12 / 5}\left\|u_{n}-u\right\|_{12 / 5}+\left\|\phi_{u_{n}}\right\|_{6}\left\|u_{n}\right\|_{6}^{\alpha}\left\|u_{n}-u\right\|_{\beta}\right]
\end{aligned}
$$

where $\beta=6 /(5-\alpha) \in(2,6)$. Then, by Lemma 2.1 and Sobolev's embedding theorem, we have

$$
\int_{\mathbb{R}^{3}} f\left(u_{n}\right) \phi_{u_{n}}\left(u_{n}-u\right) \mathrm{d} x \rightarrow 0, \quad n \rightarrow \infty .
$$

Similarly,

$$
\int_{\mathbb{R}^{3}} f(u) \phi_{u}\left(u_{n}-u\right) \mathrm{d} x \rightarrow 0, \quad n \rightarrow \infty
$$

Noting that $u_{n} \rightarrow u$ in $E$, we have that

$$
\left\langle u, u_{n}-u\right\rangle \rightarrow 0
$$

Thus,

$$
\begin{aligned}
\left\langle J_{q}^{\prime}\left(u_{n}\right)-J_{q}^{\prime}(u), u_{n}-u\right\rangle & \left(a+\left\|u_{n}\right\|^{2}\right)\left\langle u_{n}, u_{n}-u\right\rangle-\left(a+\|u\|^{2}\right)\left\langle u, u_{n}-u\right\rangle \\
= & +q \int_{\mathbb{R}^{3}}\left(f\left(u_{n}\right) \phi_{u_{n}}-f(u) \phi_{u}\right)\left(u_{n}-u\right) \mathrm{d} x \\
& -\int_{\mathbb{R}^{3}}\left(g\left(u_{n}\right)-g(u)\right)\left(u_{n}-u\right) \mathrm{d} x \\
= & \left(a+\left\|u_{n}\right\|^{2}\right)\left\|u_{n}-u\right\|^{2}+\left(\left\|u_{n}\right\|^{2}-\|u\|^{2}\right)\left\langle u, u_{n}-u\right\rangle \\
& +q \int_{\mathbb{R}^{3}}\left(f\left(u_{n}\right) \phi_{u_{n}}-f(u) \phi_{u}\right)\left(u_{n}-u\right) \mathrm{d} x \\
& -\int_{\mathbb{R}^{3}}\left(g\left(u_{n}\right)-g(u)\right)\left(u_{n}-u\right) \mathrm{d} x \\
= & \left(a+\left\|u_{n}\right\|^{2}\right)\left\|u_{n}-u\right\|^{2}+o_{n}(1), \quad n \rightarrow \infty .
\end{aligned}
$$

Consequently, we have $\left\|u_{n}-u\right\| \rightarrow 0$, that is, $u_{n} \rightarrow u$ in $E$, and the proof is complete. 
Theorem 3.4 Assume that $(f),\left(h_{1}\right),\left(h_{2}\right),\left(g_{1}\right),\left(g_{2}\right)$, and $\left(g_{3}\right)$ hold. Then, for any $q \geq 0$, there exists $u_{0} \in H$ such that

$$
J_{q}^{\prime}\left(u_{0}\right)=0 \quad \text { and } \quad J_{q}\left(u_{0}\right)=c_{0}
$$

where $c_{0}$ is given by Lemma 3.2

Proof Since $c_{0}=\inf \left\{J_{q}(u): u \in \bar{B}_{\rho}\right\}<0$, by Ekeland's variational principle [18], there exists a sequence $\left\{u_{n}\right\} \subset \bar{B}_{\rho}$ such that

$$
c_{0} \leq J_{q}\left(u_{n}\right) \leq c_{0}+\frac{1}{n}, \quad J_{q}(w) \geq J_{q}\left(u_{n}\right)-\frac{1}{n}\left\|w-u_{n}\right\|, \quad \forall w \in \bar{B}_{\rho} .
$$

By the standard procedure, we can see that $\left\{u_{n}\right\}$ is a bounded (PS) sequence of $J_{q}$. By Lemma 3.3, $\left\{u_{n}\right\}$ possesses a convergent subsequence. We may assume that, up to a subsequence, $u_{n} \rightarrow u_{0}$ in $H$. Hence $J_{q}\left(u_{0}\right)=c_{0}$ and $J_{q}^{\prime}\left(u_{0}\right)=0$.

\section{Proof of Theorem 1.1}

In this section, we aim to prove that system (1.1) has a positive energy solution. It is difficult to prove the boundedness of a (PS) sequence of $J_{q}$. Here we consider the following perturbed functional. For $u \in H$,

$$
\begin{aligned}
J_{q, \lambda}^{T}(u) & =\frac{a}{2}\|u\|^{2}+\frac{1}{4}\|u\|^{4}+\frac{q}{2} h_{T}(u) \int_{\mathbb{R}^{3}} F(u) \phi_{u} \mathrm{~d} x-\lambda \int_{\mathbb{R}^{3}} G(u) \mathrm{d} x-\int_{\mathbb{R}^{3}} h u \mathrm{~d} x \\
& =A(u)-\lambda B(u),
\end{aligned}
$$

where $\lambda \in[1 / 2,1]$ and

$$
\begin{aligned}
& A(u)=\frac{a}{2}\|u\|^{2}+\frac{1}{4}\|u\|^{4}+\frac{q}{2} h_{T}(u) \int_{\mathbb{R}^{3}} F(u) \phi_{u} \mathrm{~d} x-\int_{\mathbb{R}^{3}} h u \mathrm{~d} x, \\
& B(u)=\int_{\mathbb{R}^{3}} G(u) \mathrm{d} x .
\end{aligned}
$$

Then $J_{q, \lambda}^{T}$ is of $C^{1}$ functionals on $X=H$, and for any $u, v \in H$, we have

$$
\begin{aligned}
\left\langle\left(J_{q, \lambda}^{T}\right)^{\prime}(u), v\right\rangle= & \left(a+\|u\|^{2}+a_{q}^{T}(u)\right)\langle u, v\rangle+q h_{T}(u) \int_{\mathbb{R}^{3}} f(u) \phi_{u} v \mathrm{~d} x \\
& -\lambda \int_{\mathbb{R}^{3}} g(u) v \mathrm{~d} x-\int_{\mathbb{R}^{3}} h v \mathrm{~d} x,
\end{aligned}
$$

where

$$
a_{q}^{T}(u)=q T^{-2} \chi^{\prime}\left(T^{-2}\|u\|^{2}\right) \int_{\mathbb{R}^{3}} F(u) \phi_{u} \mathrm{~d} x .
$$

The following lemmas imply that $J_{q, \lambda}^{T}$ satisfies the conditions of Theorem 2.2.

Lemma 4.1 Assume that $(f),\left(h_{1}\right),\left(h_{2}\right),\left(g_{1}\right),\left(g_{2}\right)$, and $\left(g_{3}\right)$ hold. Then the following results hold: 
(i) There exist $r, a>0$ such that, for all $\lambda \in I,\left.J_{q, \lambda}^{T}\right|_{\|u\|=r} \geq a$.

(ii) There exists $e \in H \backslash\{0\}$ with $\|e\| \geq r$ such that, for all $\lambda \in I, J_{q, \lambda}^{T}(e)<0$.

(iii) For all $\lambda \in I$,

$$
c_{\lambda}=\inf _{\gamma \in \Gamma_{\lambda}} \max _{t \in[0,1]} J_{q, \lambda}^{T}(\gamma(t))>\max \left\{J_{q, \lambda}^{T}(0), J_{\lambda}(e)\right\}>0
$$

where

$$
\Gamma_{\lambda}=\{\gamma \in C([0,1], H): \gamma(0)=0, \gamma(1)=e\} .
$$

Proof (i) By conditions $\left(g_{1}\right)$ and $\left(g_{2}\right)$, for any $u \in H$ and $\lambda \in I$,

$$
\begin{aligned}
J_{q, \lambda}^{T}(u) & =\frac{a}{2}\|u\|^{2}+\frac{1}{4}\|u\|^{4}+\frac{q}{2} h_{T}(u) \int_{\mathbb{R}^{3}} F(u) \phi_{u} \mathrm{~d} x-\lambda \int_{\mathbb{R}^{3}} G(u) \mathrm{d} x-\int_{\mathbb{R}^{3}} h u \mathrm{~d} x \\
& \geq \frac{a}{2}\|u\|^{2}-\int_{\mathbb{R}^{3}} G(u) \mathrm{d} x-\int_{\mathbb{R}^{3}} h u \mathrm{~d} x .
\end{aligned}
$$

So, by (3.3) and Lemma 3.1, there exists $r>0$ such that, for all $\lambda \in I$ and $u \in H$ with $\|u\|=r$, we have $J_{q, \lambda}^{T}(u) \geq a>0$.

(ii) For any $\lambda \in I$, we choose a radial function $\psi \in H$ with $\psi \geq 0$ and $\|\psi\|=1$. By $\left(g_{3}\right)$, we have that, for any $C_{2}>0$ with $C_{2} \int_{\mathbb{R}^{3}} \psi^{4} \mathrm{~d} x>1 / 2$, there exists $C_{3}>0$ such that

$$
G(u) \geq C_{2}|u|^{4}-C_{3}, \quad \forall u \in \mathbb{R} .
$$

Then, for $t>T$,

$$
\begin{aligned}
J_{q, \lambda}^{T}(t \psi)= & \frac{a t^{2}}{2}\|\psi\|^{2}+\frac{t^{4}}{4}\|\psi\|^{4}+\frac{q}{2} \chi\left(\frac{t^{2}}{T^{2}}\right) \int_{\mathbb{R}^{3}} F(t \psi) \phi_{t \psi} \mathrm{d} x \\
& -\lambda \int_{\mathbb{R}^{3}} G(t \psi) \mathrm{d} x-t \int_{\mathbb{R}^{3}} h \psi \mathrm{d} x \\
= & \frac{a t^{2}}{2}+\frac{t^{4}}{4}-\lambda \int_{\mathbb{R}^{3}} G(t \psi) \mathrm{d} x-t \int_{\mathbb{R}^{3}} h \psi \mathrm{d} x \\
\leq & \frac{a t^{2}}{2}+\frac{t^{4}}{4}-\frac{1}{2} C_{2} t^{4} \int_{\mathbb{R}^{3}} \psi^{4} \mathrm{~d} x+C_{4} .
\end{aligned}
$$

Then we can choose $t>0$ large enough such that $J_{q, \lambda}^{T}(t \psi)<0$. Taking $e=t \psi$, then (ii) holds.

(iii) Now fix $\lambda \in I$ and $\gamma \in \Gamma_{\lambda}$ with $\gamma(1)=e$. By the continuity of $\gamma$, there exists $t_{\gamma} \in(0,1)$ such that $\left\|\gamma\left(t_{\gamma}\right)\right\|=r$. Therefore, for any $\lambda \in I$, we have

$$
c_{\lambda} \geq \inf _{\gamma \in \Gamma_{\lambda}} J_{q, \lambda}^{T}\left(\gamma\left(t_{\gamma}\right)\right) \geq a>0 .
$$

The proof is complete.

Lemma 4.2 For any $\lambda \in I$ and $4 q^{2} \widetilde{T}<a$, each bounded Palais-Smale sequence of the functional $J_{q, \lambda}^{T}$ admits a convergent subsequence, where $\widetilde{T}=C_{1}\left(T^{2}+T^{2 \alpha}\right)$. 
Proof Let $\lambda \in I$ and $\left\{u_{n}\right\}$ be a bounded (PS) sequence of $J_{q, \lambda}^{T}$, that is, $\left\{u_{n}\right\}$ and $J_{q, \lambda}^{T}\left(u_{n}\right)$ are bounded, $\left(J_{q, \lambda}^{T}\right)^{\prime}\left(u_{n}\right) \rightarrow 0$ in $H^{-1}$. We may assume that, up to a subsequence,

$$
\begin{array}{ll}
u_{n} \rightarrow u & \text { in } H, \\
u_{n} \rightarrow u & \text { in } L^{s}\left(\mathbb{R}^{3}\right), s \in(2,6), \\
u_{n} \rightarrow u & \text { a.e. on } \mathbb{R}^{3} .
\end{array}
$$

By (4.1) and the proof of Lemma 3.3,

$$
\begin{aligned}
& \left\langle\left(J_{q, \lambda}^{T}\right)^{\prime}\left(u_{n}\right)-\left(J_{q, \lambda}^{T}\right)^{\prime}(u), u_{n}-u\right\rangle \\
& =\left(a+\left\|u_{n}\right\|^{2}+a_{q}^{T}\left(u_{n}\right)\right)\left\langle u_{n}, u_{n}-u\right\rangle-\left(a+\|u\|^{2}+a_{q}^{T}(u)\right)\left\langle u, u_{n}-u\right\rangle \\
& \quad+q h_{T}\left(u_{n}\right) \int_{\mathbb{R}^{3}} f\left(u_{n}\right) \phi_{u_{n}}\left(u_{n}-u\right) \mathrm{d} x-q h_{T}(u) \int_{\mathbb{R}^{3}} f(u) \phi_{u}\left(u_{n}-u\right) \mathrm{d} x \\
& \quad-\lambda \int_{\mathbb{R}^{3}}\left(g\left(u_{n}\right)-g(u)\right)\left(u_{n}-u\right) \mathrm{d} x \\
& =\left(a+\left\|u_{n}\right\|^{2}+a_{q}^{T}\left(u_{n}\right)\right)\left\langle u_{n}, u_{n}-u\right\rangle+o_{n}(1),
\end{aligned}
$$

and then $\left(a+\left\|u_{n}\right\|^{2}+a_{q}^{T}\left(u_{n}\right)\right)\left\langle u_{n}, u_{n}-u\right\rangle \rightarrow 0, n \rightarrow \infty$.

When $\left\|u_{n}\right\| \leq T$, by Lemma 2.1(iv), we obtain that

$$
\left|\int_{\mathbb{R}^{3}} F\left(u_{n}\right) \phi_{u_{n}} \mathrm{~d} x\right| \leq q C_{1}\left(\left\|u_{n}\right\|^{4}+\left\|u_{n}\right\|^{2(1+\alpha)}\right)=q T^{2} \widetilde{T} .
$$

By (4.2),

$$
\left|a_{q}^{T}\left(u_{n}\right)\right| \leq q T^{-2}\left|\chi^{\prime}\left(T^{-2}\left\|u_{n}\right\|^{2}\right)\right|\left|\int_{\mathbb{R}^{3}} F\left(u_{n}\right) \phi_{u_{n}} \mathrm{~d} x\right| \leq 4 q^{2} \widetilde{T} .
$$

It follows from the assumption $4 q^{2} \widetilde{T}<a$ that $a+\left\|u_{n}\right\|^{2}+a_{q}^{T}\left(u_{n}\right) \geq a-4 q^{2} \widetilde{T}>0$, hence $\left\langle u_{n}, u_{n}-u\right\rangle \rightarrow 0$. This together with $u_{n} \rightarrow u$ shows that $u_{n} \rightarrow u$ in $H$. The proof is complete.

Lemma 4.3 Let $4 q^{2} \widetilde{T}<a$, then for almost every $\lambda \in I$, there exists $u^{\lambda} \in H \backslash\{0\}$ such that $\left(J_{q, \lambda}^{T}\right)^{\prime}\left(u^{\lambda}\right)=0$ and $J_{q, \lambda}^{T}\left(u^{\lambda}\right)=c_{\lambda}$.

Proof Firstly, it is easy to see that $B(u) \geq 0$ and $A(u) \rightarrow \infty$ as $\|u\| \rightarrow \infty$. Then, by Lemma 4.1 and Theorem 2.2, for almost every $\lambda \in I$, there exists a bounded sequence $\left\{u_{n}^{\lambda}\right\} \subset H$ such that

$$
\left(J_{q, \lambda}^{T}\right)^{\prime}\left(u_{n}^{\lambda}\right) \rightarrow 0, \quad J_{q, \lambda}^{T}\left(u_{n}^{\lambda}\right) \rightarrow c_{\lambda}
$$

By Lemma 4.2, we can obtain that there exists $u^{\lambda} \in H$ such that $u_{n}^{\lambda} \rightarrow u^{\lambda}$. Hence $\left(J_{q, \lambda}^{T}\right)^{\prime}\left(u^{\lambda}\right)=$ 0 and $J_{q, \lambda}^{T}\left(u^{\lambda}\right)=c_{\lambda}$. Furthermore, it follows from $f(t)=g(t)=0$ for $t<0$ and Lemma 4.1(i) that $u^{\lambda} \in H \backslash\{0\}$. 
By virtue of Lemma 4.3, we have that there exist $\left\{\lambda_{n}\right\} \subset I$ with $\left\{\lambda_{n}\right\} \rightarrow 1^{-}$and a nonnegative sequence $\left\{u^{\lambda_{n}}\right\}$ (denoted by $\left\{u_{n}\right\}$ for simplicity) satisfying

$$
\left(J_{q, \lambda_{n}}^{T}\right)^{\prime}\left(u_{n}\right)=0, \quad J_{q, \lambda_{n}}^{T}\left(u_{n}\right)=c_{\lambda_{n}} .
$$

In order to obtain that $\left\|u_{n}\right\| \leq T / \sqrt{2}$, the following Pohozaev identity is important, the proof can be obtained as Lemma 2.2 in [13], and the details are omitted here.

Lemma 4.4 If $u \in H$ is a weak solution of

$$
\begin{cases}{\left[a+\|u\|^{2}+a_{q}^{T}(u)\right](-\Delta u+b u)+q h_{T}(u) \phi f(u)=\lambda g(u)+h(x),} & \text { in } \mathbb{R}^{3}, \\ -\Delta \phi=2 q F(u), & \text { in } \mathbb{R}^{3},\end{cases}
$$

then the following Pohozaev identity holds:

$$
\begin{aligned}
{[a} & \left.+\|u\|^{2}+a_{q}^{T}(u)\right]\left(\frac{1}{2} \int_{\mathbb{R}^{3}}|\nabla u|^{2} \mathrm{~d} x+\frac{3}{2} b \int_{\mathbb{R}^{3}} u^{2} \mathrm{~d} x\right)+\frac{5}{2} q h_{T}(u) \int_{\mathbb{R}^{3}} F(u) \phi_{u} \mathrm{~d} x \\
& =3 \lambda \int_{\mathbb{R}^{3}} G(u) \mathrm{d} x+\int_{\mathbb{R}^{3}}(3 h(x)+\langle\nabla h(x), x\rangle) u \mathrm{~d} x .
\end{aligned}
$$

The following lemma shows that $\left\|u_{n}\right\| \leq T / \sqrt{2}$.

Lemma 4.5 Let $u_{n}$ be a critical point of $J_{q, \lambda_{n}}^{T}$ at level $c_{\lambda_{n}}$. Then there exist $T_{0}>1$ and $q_{0}>0$ with $16 q_{0}^{2} T_{0}^{2} \widetilde{T}_{0}<$ a such that, for any $q \in\left[0, q_{0}\right)$ and $b \leq 1 / 4 \gamma_{2}^{2},\left\|u_{n}\right\| \leq T_{0} / \sqrt{2}$ for all $n \in \mathbb{N}$.

Proof We will argue by contradiction. Assume that for every $T>1$ there exists $q_{T}$ satisfying $16 q_{T}^{2} T^{2} \widetilde{T}<a$ such that

$$
\limsup _{n \rightarrow \infty}\left\|u_{n}\right\| \geq T / \sqrt{2}
$$

Firstly, since $\left(J_{q, \lambda_{n}}^{T}\right)^{\prime}\left(u_{n}\right)=0$, by (4.7), $u_{n}$ satisfies the following Pohozaev identity:

$$
\begin{aligned}
{[a+} & \left.\left\|u_{n}\right\|^{2}+a_{q}^{T}\left(u_{n}\right)\right]\left(\frac{1}{2} \int_{\mathbb{R}^{3}}\left|\nabla u_{n}\right|^{2} \mathrm{~d} x+\frac{3}{2} b \int_{\mathbb{R}^{3}} u_{n}^{2} \mathrm{~d} x\right) \\
& +\frac{5}{2} q h_{T}\left(u_{n}\right) \int_{\mathbb{R}^{3}} F\left(u_{n}\right) \phi_{u_{n}} \mathrm{~d} x \\
= & 3 \lambda_{n} \int_{\mathbb{R}^{3}} G\left(u_{n}\right) \mathrm{d} x+\int_{\mathbb{R}^{3}}(3 h(x)+\langle\nabla h(x), x\rangle) u_{n} \mathrm{~d} x .
\end{aligned}
$$

By using $J_{q, \lambda_{n}}^{T}\left(u_{n}\right)=c_{\lambda_{n}}$, we have that

$$
\begin{aligned}
& \frac{3 a}{2}\left\|u_{n}\right\|^{2}+\frac{3}{4}\left\|u_{n}\right\|^{4}+\frac{3 q}{2} h_{T}\left(u_{n}\right) \int_{\mathbb{R}^{3}} F\left(u_{n}\right) \phi_{u_{n}} \mathrm{~d} x-3 \lambda_{n} \int_{\mathbb{R}^{3}} G\left(u_{n}\right) \mathrm{d} x-3 \int_{\mathbb{R}^{3}} h u_{n} \mathrm{~d} x \\
& \quad=3 c_{\lambda_{n}} .
\end{aligned}
$$


Chi Boundary Value Problems

(2019) 2019:139

Page 13 of 16

Hence, by (4.5), (4.9), (4.10), and $\left(h_{3}\right)$, we can obtain that

$$
\begin{aligned}
\left(\left\|u_{n}\right\|^{2}+\frac{a}{2}\right) \int_{\mathbb{R}^{3}}\left|\nabla u_{n}\right|^{2} \mathrm{~d} x \\
\leq \\
\left.\leq a+\left\|u_{n}\right\|^{2}+a_{q}^{T}\left(u_{n}\right)\right] \int_{\mathbb{R}^{3}}\left|\nabla u_{n}\right|^{2} \mathrm{~d} x \\
=3 c_{\lambda_{n}}+\frac{3}{2} a_{q}^{T}\left(u_{n}\right)\left\|u_{n}\right\|^{2}+\frac{3}{4}\left\|u_{n}\right\|^{4}+q h_{T}\left(u_{n}\right) \int_{\mathbb{R}^{3}} F\left(u_{n}\right) \phi_{u_{n}} \mathrm{~d} x \\
\quad-\int_{\mathbb{R}^{3}}\langle\nabla h(x), x) u_{n} \mathrm{~d} x \\
\leq 3 c_{\lambda_{n}}+\frac{3}{2} a_{q}^{T}\left(u_{n}\right)\left\|u_{n}\right\|^{2}+\frac{3}{4}\left\|u_{n}\right\|^{4}+q h_{T}\left(u_{n}\right) \int_{\mathbb{R}^{3}} F\left(u_{n}\right) \phi_{u_{n}} \mathrm{~d} x .
\end{aligned}
$$

Now we estimate the right-hand side of (4.11), by Lemma 4.1 and (4.3), we have

$$
\begin{aligned}
c_{\lambda_{n}} & \leq \max _{t} J_{q, \lambda_{n}}^{T}(t \psi) \\
& \leq \max _{t}\left\{\frac{a t^{2}}{2}+\frac{t^{4}}{4}-\lambda_{n} \int_{\mathbb{R}^{3}} G(t \psi) \mathrm{d} x\right\}+\max _{t} \frac{q}{2} \chi\left(\frac{t^{2}}{T^{2}}\right) \int_{\mathbb{R}^{3}} F(t \psi) \phi_{t \psi} \mathrm{d} x \\
& \leq \max _{t}\left\{\frac{a t^{2}}{2}+\frac{t^{4}}{4}-\frac{1}{2} C_{2} t^{4} \int_{\mathbb{R}^{3}} \psi^{4} \mathrm{~d} x+C_{4}\right\}+A_{q}(T) \\
& =C_{5}+A_{q}(T) .
\end{aligned}
$$

If $t \geq T$, then $\chi\left(\frac{t^{2}}{T^{2}}\right)=0$. Thus, by (4.4), we have that

$$
A_{q}(T) \leq \frac{q}{2} \max _{t \in[0, T]}\left|\int_{\mathbb{R}^{3}} F(t \psi) \phi_{t \psi} \mathrm{d} x\right| \leq \frac{1}{2} q^{2} T^{2} \widetilde{T} .
$$

By (4.4) and (4.5), we also have that

$$
q h_{T}\left(u_{n}\right) \int_{\mathbb{R}^{3}} F\left(u_{n}\right) \phi_{u_{n}} \mathrm{~d} x \leq q^{2} T^{2} \widetilde{T}
$$

and

$$
\left|a_{q}^{T}\left(u_{n}\right)\right|\left\|u_{n}\right\|^{2} \leq 4 q^{2} T^{2} \widetilde{T} .
$$

Then, by (4.11), we can obtain that

$$
\left(\left\|u_{n}\right\|^{2}+\frac{3 a}{4}\right) \int_{\mathbb{R}^{3}}\left|\nabla u_{n}\right|^{2} \mathrm{~d} x \leq \frac{3}{4}\left\|u_{n}\right\|^{4}+3\left(C_{5}+\frac{1}{2} q^{2} T^{2} \widetilde{T}\right)+6 q^{2} T^{2} \widetilde{T}+q^{2} T^{2} \widetilde{T},
$$

which implies that

$$
\left(\frac{1}{4}\left\|u_{n}\right\|^{2}+\frac{3 a}{4}\right) \int_{\mathbb{R}^{3}}\left|\nabla u_{n}\right|^{2} \mathrm{~d} x \leq \frac{3 b}{4}\left\|u_{n}\right\|^{2} \int_{\mathbb{R}^{3}} u_{n}^{2} \mathrm{~d} x+3 C_{5}+\frac{17}{2} q^{2} T^{2} \widetilde{T} .
$$

Since $b \leq 1 / 4 \gamma_{2}^{2}$, (4.12) implies that

$$
\frac{3 a}{4} \int_{\mathbb{R}^{3}}\left|\nabla u_{n}\right|^{2} \mathrm{~d} x \leq 3 C_{5}+\frac{17}{2} q^{2} T^{2} \widetilde{T} .
$$


On the other hand, since $\left\langle\left(J_{q, \lambda_{n}}^{T}\right)^{\prime}\left(u_{n}\right), u_{n}\right\rangle=0$, by $(4.1),\left(g_{1}\right)$, and $\left(g_{2}\right)$, we have that

$$
\begin{aligned}
(a & \left.+\left\|u_{n}\right\|^{2}+a_{q}^{T}\left(u_{n}\right)\right)\left\|u_{n}\right\|^{2}+q h_{T}\left(u_{n}\right) \int_{\mathbb{R}^{3}} f\left(u_{n}\right) \phi_{u_{n}} n_{n} \mathrm{~d} x \\
& =\lambda_{n} \int_{\mathbb{R}^{3}} g\left(u_{n}\right) n_{n} \mathrm{~d} x+\int_{\mathbb{R}^{3}} h u_{n} \mathrm{~d} x \\
& \leq \frac{a}{2 \gamma_{2}^{2}}\left\|u_{n}\right\|_{2}^{2}+C\left\|u_{n}\right\|_{6}^{6}+\gamma_{2}\|h\|_{2}\left\|u_{n}\right\| .
\end{aligned}
$$

Thus, by (4.12) and (4.13), we obtain that

$$
\frac{3 a}{4}\left\|u_{n}\right\|^{2}-\gamma_{2}\|h\|_{2}\left\|u_{n}\right\| \leq C\left\|u_{n}\right\|_{6}^{6} \leq S^{-3} C\left(4 C_{5} / a+\frac{34}{3 a} q^{2} T^{2} \widetilde{T}\right)^{3}
$$

Since $\gamma_{2}\|h\|_{2}<\eta(\rho)$ in Lemma 3.1 and $16 q_{T}^{2} T^{2} \widetilde{T}<a$, then we have

$$
\frac{3 a}{4}\left\|u_{n}\right\|^{2}-\eta(\rho)\left\|u_{n}\right\|<S^{-3} C\left(4 C_{5} / a+17 / 24\right)^{3} .
$$

By (4.8) and (4.14), it is impossible for $T>1$ large enough. Thus, we obtain the conclusion.

Proof of Theorem 1.1 By virtue of the result of Theorem 3.4, in order to prove Theorem 1.1, here we just need to prove that problem (1.1) has a positive radial solution $v_{0}$ with positive energy. Let $T_{0}, q_{0}$ be defined as in Lemma 4.5 and $u_{n}$ be a critical point of $J_{q, \lambda_{n}}^{T_{0}}$ at level $c_{\lambda_{n}}$. From Lemma 4.5 we have that $\left\|u_{n}\right\| \leq T_{0} / \sqrt{2}$ and $\left\{c_{\lambda_{n}}\right\}$ is bounded. So

$$
\begin{aligned}
J_{q, \lambda_{n}}^{T_{0}}\left(u_{n}\right)= & \frac{a}{2}\left\|u_{n}\right\|^{2}+\frac{1}{4}\left\|u_{n}\right\|^{4}+\frac{q}{2} h_{T}\left(u_{n}\right) \int_{\mathbb{R}^{3}} F\left(u_{n}\right) \phi_{u_{n}} \mathrm{~d} x \\
& -\lambda_{n} \int_{\mathbb{R}^{3}} G\left(u_{n}\right) \mathrm{d} x-\int_{\mathbb{R}^{3}} h u_{n} \mathrm{~d} x .
\end{aligned}
$$

Now we claim that $\left\{u_{n}\right\}$ is a (PS) sequence of $J_{q}$. Indeed,

$$
J_{q}\left(u_{n}\right)=J_{q, \lambda_{n}}^{T_{0}}\left(u_{n}\right)+\left(\lambda_{n}-1\right) \int_{\mathbb{R}^{3}} G\left(u_{n}\right) \mathrm{d} x
$$

and

$$
\left\langle J_{q}^{\prime}\left(u_{n}\right), v\right\rangle=\left\langle\left(J_{q, \lambda_{n}}^{T_{0}}\right)^{\prime}\left(u_{n}\right), v\right\rangle+\left(\lambda_{n}-1\right) \int_{\mathbb{R}^{3}} g\left(u_{n}\right) v \mathrm{~d} x .
$$

The fact $\left\{u_{n}\right\}$ is bounded implies that $\int_{\mathbb{R}^{3}} G\left(u_{n}\right) \mathrm{d} x$ is bounded and $\left\{\int_{\mathbb{R}^{3}} g\left(u_{n}\right) v \mathrm{~d} x\right\} \leq C\|v\|$. Thus, when $\lambda_{n} \rightarrow 1$, we have that $\left\{u_{n}\right\}$ is a bounded (PS) sequence of $J_{q}$. By Lemma 3.3, $\left\{u_{n}\right\}$ has a convergent subsequence, we may assume that $u_{n} \rightarrow v_{0}$. Consequently, $J_{q}^{\prime}\left(v_{0}\right)=0$. According to Lemma 4.1, we have that

$$
J_{q}\left(v_{0}\right)=\lim _{n \rightarrow \infty} J_{q}\left(u_{n}\right)=\lim _{n \rightarrow \infty} J_{q, \lambda_{n}}^{T_{0}}\left(u_{n}\right) \geq c>0,
$$

and $v_{0}$ is a positive solution by the condition of $f(t)=g(t)=0$ for $t<0$. Thus, the proof is finished. 


\section{Acknowledgements}

The author would like to express sincere thank to the anonymous referee and the handling editor whose valuable comments greatly improve the manuscript.

\section{Funding}

The work was supported by the National Natural Science Foundation of China (Grant No. 11801160) and the Natural Science Foundation of Hunan Province (Grant No. 2019JJ50096).

\section{Availability of data and materials}

Not applicable.

\section{Competing interests}

The author declares that she has no competing interests.

\section{Authors' contributions}

The author read and approved the final manuscript.

\section{Publisher's Note}

Springer Nature remains neutral with regard to jurisdictional claims in published maps and institutional affiliations.

Received: 26 March 2019 Accepted: 13 August 2019 Published online: 27 August 2019

\section{References}

1. Alvês, C.O., Correa, F.J.S.A., Ma, T.F.: Positive solutions for a quasilinear elliptic equation of Kirchhoff type. Comput. Math. Appl. 49, 85-93 (2005)

2. Avci, M., Cekic, B., Mashiyev, R.A.: Existence and multiplicity of the solutions of the $p(x)$-Kirchhoff type equation via genus theory. Math. Methods Appl. Sci. 34(14), 1751-1759 (2011)

3. Azzollini, A., d'Avenia, P.: On a system involving a critically growing nonlinearity. J. Math. Anal. Appl. 387(1), 433-438 (2012)

4. Azzollini, A., d'Avenia, P., Luisi, V.: Generalized Schrödinger-Poisson type system. arXiv:1009.2728v3

5. Benci, V., Fortunato, D.: An eigenvalue problem for the Schrödinger-Maxwell equations. Topol. Methods Nonlinear Anal. 11, 283-293 (1998)

6. Cheng, B., Wu, X.: Existence results of positive solutions of Kirchhoff type problems. Nonlinear Anal. 71, 4883-4892 (2009)

7. Duan, L., Huang, L.H.: Infinitely many solutions for sublinear Schrödinger-Kirchhoff-type equations with genera potentials. Results Math. 66, 181-197 (2014)

8. He, X., Zou, W.: Infinitely many positive solutions for Kirchhoff-type problems. Nonlinear Anal. 70, 1407-1414 (2009)

9. He, X., Zou, W.: Existence and concentration behavior of positive solutions for a Kirchhoff equation in $\mathbb{R}^{3}$. J. Differ. Equ. $252,1813-1834(2012)$

10. Jeanjean, L.: On the existence of bounded Palais-Smale sequences and application to a Landesman-Lazer-type problem set on $\mathbb{R}^{N}$. Proc. R. Soc. Edinb., Sect. A 129(4), 787-809 (1999)

11. Jeanjean, L., Le Coz, S.: An existence and stability result for standing waves of nonlinear Schrödinger equations. Adv. Differ. Equ. 11(7), 813-840 (2006)

12. Kirchhoff, G.: Mechanik. Teubner, Leipzig (1883)

13. Li, F., Zhang, Q.: Existence of positive solutions to the Schrödinger-Poisson system without compactness conditions. J. Math. Anal. Appl. 401, 754-762 (2013)

14. Li, Y., Li, F., Shi, J.: Existence of a positive solution to Kirchhoff type problems without compactness conditions. J. Differ Equ. 253, 2285-2294 (2012)

15. Liu, W., He, X.: Multiplicity of high energy solutions for superlinear Kirchhoff equations. J. Appl. Math. Comput. 39 473-487 (2012)

16. Pankov, A.: Periodic nonlinear Schrödinger equation with application to photonic crystals. Milan J. Math. 73, 259-287 (2005)

17. Perera, K., Zhang, Z.: Nontrivial solutions of Kirchhoff-type problems via the Yang index. J. Differ. Equ. 221(1), 246-255 (2006)

18. Struwe, M.: Variational Methods: Applications to Nonlinear Partial Differential Equations and Hamiltonian Systems Springer, Berlin (1990)

19. Sun, J.: Infinitely many solutions for a class of sublinear Schrödinger-Maxwell equations. J. Math. Anal. Appl. 390, 514-522 (2012)

20. Sun, J., Chen, H., Nieto, J.J.: On ground state solutions for some non-autonomous Schrödinger-Poisson systems. J. Differ. Equ. 252, 3365-3380 (2012)

21. Sun, J., Chen, H., Yang, L.: Positive solutions of asymptotically linear Schrödinger-Poisson systems with a radial potential vanishing at infinity. Nonlinear Anal. 74, 413-423 (2011)

22. Sun, J., Liu, S.: Nontrivial solutions of Kirchhoff type problems. Appl. Math. Lett. 25, 500-504 (2012)

23. Sun, J., Wu, T.-F.: Ground state solutions for an indefinite Kirchhoff type problem with steep potential well. J. Differ Equ. 256, 1771-1792 (2014)

24. Szulkin, A., Weth, T.: Ground state solutions for some indefinite variational problems. J. Funct. Anal. 257, 3802-3822 (2009)

25. Tang, X.H.: New super-quadratic conditions on ground state solutions for superlinear Schrödinger equation. Adv. Nonlinear Stud. 14, 349-361 (2014)

26. Willem, M.: Minimax Theorems. Birkhäuser, Boston (1996) 
27. $W u, X$ : Existence of nontrivial solutions and high energy solutions for Schrödinger-Kirchhoff-type equations in $\mathbb{R}^{N}$. Nonlinear Anal., Real World Appl. 12, 1278-1287 (2011)

28. Xiang, M.Q., Rădulescu, V.D., Zhang, B.: Fractional Kirchhoff problems with critical Trudinger-Moser nonlinearity. Calc. Var. Partial Differ. Equ. 58, 57 (2019) https://doi.org/10.1007/s00526-019-1499-y

29. Xiang, M.Q., Rădulescu, V.D., Zhang, B.: Nonlocal Kirchhoff diffusion problems: local existence and blow-up of solutions. Nonlinearity 31, 3228-3250 (2018)

30. Xiang, M.Q., Rădulescu, V.D., Zhang, B.: Combined effects for fractional Schrödinger-Kirchhoff systems with critical nonlinearities. ESAIM Control Optim. Calc. Var. 24, 1249-1273 (2018)

31. Zhang, Q., Li, F., Liang, Z.: Existence of multiple positive solutions to nonhomogeneous Schrödinger-Poisson system. Appl. Math. Comput. 259, 353-363 (2015)

32. Zhao, G., Zhu, X., Li, Y.: Existence of infinitely many solutions to a class of Kirchhoff-Schrödinger-Poisson system. Appl. Math. Comput. 256, 572-581 (2015)

Submit your manuscript to a SpringerOpen ${ }^{\circ}$ journal and benefit from:

- Convenient online submission

- Rigorous peer review

Open access: articles freely available online

- High visibility within the field

- Retaining the copyright to your article

Submit your next manuscript at $\boldsymbol{\nabla}$ springeropen.com 